\title{
Dose-volume histogram constrains for small intestine in postoperative transcutaneous radiotherapy of endometrial carcinoma: comparison between conventional and conformal techniques
}

\author{
Anela Ramić1* , Dženita Ljuca², Goran Marosević ${ }^{1}$
}

'Department of Radiotherapy, Clinic for Oncology, Hematology and Radiotherapy and ${ }^{2}$ Gynecology and Obstetrics Clinic, University Clinical Center, Trnovac bb, 75000 Tuzla, Bosnia and Herzegovina

\begin{abstract}
Introduction: The aim of this study was to determine the dose-volume histogram (DVH) constrains of conventional and conformal transcutaneous radiotherapy for small intestine and perform their comparison.

Methods: This retrospective-prospective study included patients who were treated for endometrial cancer using conventional transcutaneous radiotherapy at the Department of Radiotherapy Clinic of Oncology, Hematology and Radiotherapy, University Clinical Center Tuzla in the period from 2009 to 2011. The study was performed on patients of all ages suffering from this condition. The study involved 35 patients. DVH parameters which were analyzed are: minimum dose $\left(D_{\min }\right)$, maximum dose $\left(D_{\max }\right)$, medium dose $\left(D_{\text {aver }}\right)$ of the small intestine, as well as the volume of the small intestine, which is included in $75 \%, 95 \%$ and $100 \%$ dose $\left(V_{33,75 G y^{\prime}} V_{42,75 G y^{\prime}} V_{45 G y}\right)$ expressed in percentages and cubic centimeters of the affected organ. Working hypothesis was tested with paired $t$ test. The difference between the variables at the level of $p<0.05$ was considered statistically significant.
\end{abstract}

Results: DVH constrains of transcutaneous conformal radiotherapy showed significantly smaller dose contribution on small intestine than DVH parameters of conventional transcutaneous radiotherapy ( $p$ $<0.0001$ ).

Conclusion: The dose contribution on small intestine was significantly lower by planning three-dimensional conformal transcutaneous radiotherapy in comparison to the conventional planning.

Keywords: transcutaneous postoperative radiotherapy, endometrial cancer

\footnotetext{
*Corresponding author: Anela Ramić, Department of Radiotherapy, Clinic for Oncology, Hematology and Radiotherapy, University Clinical Center, Trnovac bb, 75000 Tuzla, Bosnia and Herzegovina Phone: +38761109766

Email: neca_kurtovic@yahoo.com
}

Submitted 21 March 2013 / Accepted 7 May 2013

\section{INTRODUCTION}

In developed countries, thanks to early detection in noninvasive stage, endometrial cancer is the most common malignant tumor of the female genital organs (1). The application of radiotherapy in the treatment of endometrial cancer is often used be- 
cause the results of a combination of surgical treatment and radiotherapy are often better than surgical treatment only (2). Indications for use of radiotherapy depend on the type and the degree of malignant disease spread, the clinical condition of the patient and the use of other therapeutic modalities (3). Radiation planning includes the possibility of objective quantification of basic planning procedures, which require the application of methods, which in the process of creating treatment plan, take into account the requirements and expectations of radiotherapists and physicists, the definition of anatomical structures on the basis of patient series CT / MR images, and interactive / or automated optimization desired combination of air beams for better dose distribution, the possibility of checking the reliability of the data entered, ensuring reproducibility and precision radiation therapy of patients under the same conditions as prescribed by the given plan. Conventional treatment is based on radiation with two to four coplanar air fields, whose parameters are determined by standard simulation scopy until the estimation of isodose distribution is performed on the basis of body contours and organic structures on one "reference" CT section. 2D radiotherapy planning is still a standard that is applied in a number of radiotherapy institutions in addition to its limits and disadvantages. The belief that technological advances in medicine must lead to improved quality and results of treatment, can sometimes lead to compromising the particular method. Accordingly, the present opinion is that we are planning and implementing $3 \mathrm{D}$ conformal radiotherapy closer to achieving maximum basic objectives of radiotherapy (application of cancercid dose of radiation on the tumor with the maximum preservation of the surrounding healthy tissue), but also the opinion that the use of such expensive and sophisticated technology allows only informative view of anatomical structures of interest which does not result in improving the quality of planning and implementation of radiotherapy in total (4). Quantitative evaluation of planning of transcutaneous radiotherapy using dose-volume histogram (DVH) is the basis for the selection of the optimal radiotherapy plan in order for tumor to receive the optimal dose sparing surrounding healthy tissue. The DVH is used to display the dose distribution. It is particularly useful in the evaluation of dose distribution in case we have more radiotherapy plans. DVH shows a complete dose distribution in radiated area, i.e. the amount of dose that is delivered to the target volume and organs at risk (5). The aim of this study is to determine the DVH parameters of conventional and conformal transcutaneous radiotherapy for small intestine, and make a comparison of these parameters.

\section{METHODS}

\section{Patients}

In this retrospective-prospective study patients were treated for endometrial cancer using conventional transcutaneous radiotherapy. All patients were treated at the Department of Radiotherapy Clinic of Oncology, Hematology and Radiotherapy, University Clinical Center Tuzla in the period from 2009 to 2011. The study was performed on patients of all ages suffering from this condition. The study involved 35 patients. The control group consisted of plans for patients treated with conventional transcutaneous radiotherapy. Three-dimensional conformal radiotherapy planning was made for the same patients, and these plans were also included in the experimental group. Including factors: patients suffering from endometrial carcinoma treated with conventional transcutaneous radiotherapy. Excluding factors were: patients suffering from endometrial cancer who were not treated with conventional transcutaneous radiotherapy. Data on patients treated for endometrial cancer are taken from ImpacMosaiq software system for storing data in radiotherapy. Planning a three-dimensional conformal radiotherapy was performed on the FOCAL and Xio planning system for radiotherapy on which planning was previously done for conventional transcutaneous radiotherapy.

\section{Procedures}

Patients in the control group were treated using conventional transcutaneous radiotherapy technique with AP / PA pelvic fields. At the same CT topograms, on which the conventional radiotherapy was planned, 3D conformal radiotherapy planning was done which formed working group. 3D plan considered contouring the target volume and organs at risk (bladder, rectum and small intestine). It showed up in FOCAL system for planning radiotherapy. Delineation of the rectum, bladder and small intestine was performed on each CT section for rectum at 1 
TABLE1. DVH parameters for small intestine in conventional and conformal technique of irradiation

\begin{tabular}{|c|c|c|c|c|c|c|c|}
\hline \multirow{2}{*}{$\begin{array}{l}\text { DVH parameters for } \\
\text { small intestine }\end{array}$} & \multicolumn{3}{|c|}{ Conventional plans (35 patients) } & \multicolumn{3}{|c|}{ Conformal plans (35 patients) } & \multirow[b]{2}{*}{$p$} \\
\hline & mean $(\sigma)$ & Mean & range & mean $(\sigma)$ & Mean & range & \\
\hline $\mathrm{D}_{\min }$ & $0,84(0,36)$ & 0,83 & $(0-1,89)$ & $2,67(1,40)$ & 2,35 & $(0,92-6,55)$ & $<0.0001$ \\
\hline$D_{\max }$ & $49,06(1,32)$ & 48,96 & $(47,05-54,19)$ & $47,97(0,94)$ & 47,83 & $(46,08-50,01)$ & $<0.0001$ \\
\hline $\mathrm{D}_{\text {aver }}$ & $40,35(3,90)$ & 40,31 & $(27,21-48,30)$ & $35,77(3,87)$ & 36,09 & $(26,37-43,52)$ & $<0.0001$ \\
\hline $\mathrm{V}_{33,75 \mathrm{~Gy}}$ & $83,57(8,76)$ & 83,89 & $(54,38-96,14)$ & $56,58(15,41)$ & 55,72 & $(15,46-93,51)$ & $<0.0001$ \\
\hline$V_{42,75 G y}$ & $79,5(9,12)$ & 80,31 & $(51,29-95,14)$ & $41,74(14,77)$ & 40,67 & $(7,79-80,59)$ & $<0.0001$ \\
\hline$V_{45 G y}$ & $64,11(12,48)$ & 64,22 & $(37,69-93,44)$ & $30,20(15,97)$ & 30,77 & $(0,15-71,07)$ & $<0.0001$ \\
\hline $\mathrm{V}_{33,75 \mathrm{~Gy}}\left(\mathrm{~cm}^{3}\right)$ & $638,19(183,82)$ & 627,21 & $(338,08-1082,1)$ & $421,81(143,6)$ & 419,13 & $(180,11-840,49)$ & $<0.0001$ \\
\hline $\mathrm{V}_{42,75 \mathrm{~Gy}}\left(\mathrm{~cm}^{3}\right)$ & $606,41(174,07)$ & 596,79 & $(325,71-1016,18)$ & $311,64(126,85)$ & 309,84 & $(90,64-630,85)$ & $<0.0001$ \\
\hline $\mathrm{V}_{45 G \mathrm{y}}\left(\mathrm{cm}^{3}\right)$ & $491,6(166,84)$ & 474,28 & $(214,52-785,74)$ & $224,74(124,05)$ & 194,62 & $(0,87-569,47)$ & $<0.0001$ \\
\hline
\end{tabular}

Legend: mean - average mean

$\sigma$ - standard deviation

$\mathrm{cm}$ from the anus all the way to the rectosigmoidal crossing of the entire thickness of the wall of organ, bladder outer contour of the entire volume of organs, and the outer contour of the small intestine volume in organs that are in the field of radiation (1). Then, DVH for conventional plans was calculated on the Xio system for calculating the dose distributions and the obtained parameters consisted the control group. The amount of transcutaneous dose that organs at risk received based on planning for conventional transcutaneous radiotherapy was examined. For the same patients, after the organs at risk were contoured the target volume was contoured, too. The target volume included clinical target volume (CTV) and planning target volume (PTV). CTV covered dock uterine, vaginal scar, and regional lymph nodes, depending on the seat of primary tumor and $\mathrm{N}$ stage. CTV was shown as a margin of $7 \mathrm{~mm}$ around vascular structures. Then, on $10 \mathrm{~mm}$ around the CTV, PTV that represents the safety margin in which the error during patient repositioning and physiological organ motion (6) was included, was shown. The geometry of new conformal fields was determined and three-dimensional dose distribution was calculated on Xio system. New dose-volume histograms for organs at risk were calculated for conformal plans. We examined the amount of transcutaneous dose organs at risk received by planning based on the plan for threedimensional transcutaneous radiotherapy. From DVH parameters, the following were analyzed: the minimum dose $\left(\mathrm{D}_{\min }\right)$, maximum dose $\left(\mathrm{D}_{\max }\right)$, mean dose $\left(\mathrm{D}_{\text {aver }}\right)$ organs of the risks, as well as the volume of organs at risks included in 75\%, 95\% and 100\% dose $\left(\mathrm{V}_{33,75 \mathrm{~Gy}}, \mathrm{~V}_{42,75 \mathrm{~Gy}}, \mathrm{~V}_{45 \mathrm{~Gy}}\right)$ expressed in percentages and cubic centimeters of the affected organs.

\section{Statistical Analysis}

In the descriptive analysis, we used the arithmetic mean as a complete mean and a median as positional average. Also, along with the arithmetic mean, it was necessary to calculate the absolute measure of dispersion, which we presented as a standard deviation in order to evaluate the average deviation of a numerical characteristic of their arithmetic mean. Numerical range of features from minimum to maximum is shown along with the median. Working hypothesis was tested with paired $\mathrm{t}$ test in order to compare the DVH parameters organs at risk among treated groups. The difference between the variables at the level of $\mathrm{p}<0.05$ was considered statistically significant. ArcusQuickstat Statistical software (version 1.0.0.88, Medical Computing.) was used for data analysis.

\section{RESULTS}

In this study, DVH parameter plans for radiation for 35 patients suffering from endometrial cancer, who were treated with conventional transcutaneous radiotherapy at the Department of Radiotherapy Clinic for Oncology, Hematology and Radiotherapy in the period from 2009 to 2011 were monitored. The analyzed DVH parameters for small intestine for 35 conventional and 35 conformal radiation plans are shown in Table 1. 
DVH parameters for transcutaneous conformal radiotherapy show significantly smaller contribution dose on small intestine than DVH parameters for conventional transcutaneous radiotherapy ( $\mathrm{p}$ $<0.0001$ ). Analysis of the results showed that the $\mathrm{D}_{\min }$ is significantly lower when using conventional techniques of radiation (0.84 Gyvs2.67 Gy, p $<0.0001)$. Comparing the values of the parameters for $\mathrm{D}_{\text {max }}$ a statistically significant lower dose can be seen if conformal radiotherapy technique is applied (47.97 Gy versus 49.06 Gy, p <0.0001).

Comparing conventional and conformal radiation technique, it was observed that $\mathrm{D}_{\text {averis }}$ significantly smaller when using conformal radiation techniques (35.77 Gy versus 40.35 Gy, p <0.0001). Analyzing the DVH parameters for conventional and conformal radiation technique it is proven that the volume of small intestine covered by $75 \% \operatorname{dose}\left(\mathrm{V}_{33,75 \mathrm{~Gy}}\right)$ is statistically significantly lower if using conformal techniques $(56.58 \%$ versus $83.57 \%, \mathrm{p}<0.0001)$.

Comparison of values for the volume of the small intestine which is covered by $95 \%$ of the dose $\left(\mathrm{V}_{42,75 \mathrm{~Gy}}\right)$ showed that this volume of the small intestine is significantly smaller if using conformal radiation techniques $(41.74 \%$ versus $79.5 \%, \mathrm{p}<0.0001$ ). Volume of the small intestine which is covered by $100 \%$ of the dose $\left(\mathrm{V}_{45 \mathrm{~Gy}}\right)$ was significantly smaller if using conformal radiation techniques $(30.2 \%$ versus 64.11\%, p <0.0001).

The results for the volume of the small intestine which is covered by the $75 \%$ of the dose $\left(\mathrm{V}_{33,75 \mathrm{~Gy}}\right)$ , what is expressed in cubic centimeters, show that this volume of the small intestine is significantly lower when using conformal radiation techniques $\left(421.81 \mathrm{~cm}^{3}\right.$ versus $\left.638.19 \mathrm{~cm}^{3} \mathrm{p}<0.0001\right)$. Comparing the results for the volumes of small intestine covered by $95 \%$ and $100 \%$ of the dose $\left(\mathrm{V}_{42,75 \mathrm{~Gy}}\right.$ and $\mathrm{V}_{45 \mathrm{~Gy}}$ ), which in this case are expressed in cubic centimeters, show that both of the above volumes are significantly lower if using conformal radiation techniques $\left(311.64 \mathrm{~cm}^{3}\right.$ versus $606.41 \mathrm{~cm}^{3}$, p $<0.0001 ; 224.74 \mathrm{~cm}^{3}$ vs. $\left.491.6 \mathrm{~cm}^{3}, \mathrm{p}<0.0001\right)$.

\section{DISCUSSION}

Adjuvant radiotherapy is an important factor for loco regional control of endometrial cancer. However, applying a therapeutic dose to the target volume is limited by threshold of radio sensibility surround- ing healthy tissue and organs. Therefore, modern radiotherapy gives special attention to the doses received by the volume of risk organs (small intestine, bladder, colon), so as to reduce the risk of post radiation complications to minimum. For each organ at risk there is a maximum dose that organ may receive in the course of radiation treatment. For the small intestine it is recommended that the volume receiving $45 \mathrm{~Gy}\left(\mathrm{~V}_{45 \mathrm{GY}}\right)$ should be less than $195 \mathrm{~cm}^{3}$ (7).

This study compared the conventional and conformal radiation technique following and comparing DVH of these techniques. Our results show that the contribution of dose to the small intestine was significantly lower if conformal radiotherapy technique is applied in comparison to the conventional one. Maximum and mean dose $\left(\mathrm{D}_{\max }\right.$ and $\left.\mathrm{D}_{\text {aver }}\right)$ for the small intestine were significantly smaller if conformal radiotherapy technique is applied. The only parameter that was significantly smaller when the conventional techniques was used, was $\mathrm{D}_{\text {min }}$. Conformal techniques from multiple fields provide better coverage of therapeutic dose with target volume and better sparing of organs at risk from high doses of radiation. However, a bigger number of fields give a stronger minimum dose to a larger volume of surrounding organs (such as the small intestine). The volumes of the small intestine that are covered by $75 \%, 95 \%$ and $100 \%$ dose were significantly lower when conformal techniques were used. The reason for this is because $3 \mathrm{D}$ planning gets a complete presentation of tumor volume and surrounding structures, based on which the optimum angle of the air burst and the shape of air field are determined, which ensures maximum possible exclusion of organs at risk from the air volume, which again significantly reduces the volume of organs at risk covered by transcutaneous radiotherapy dose.

In the study Heron et al.(8) compared 3D conformal radiotherapy techniques with other radiation techniques in which DVH parameters for plans were monitored and compared. Particular attention was focused on the volume of organs at risk that received $30 \mathrm{~Gy}\left(\mathrm{~V}_{30 \mathrm{~Gy}}\right)$ as well as the volume of organs at risk that received $45 \mathrm{~Gy}\left(\mathrm{~V}_{45 \mathrm{~Gy}}\right) . \mathrm{V}_{30 \mathrm{~Gy}}$ for small intestine was $30.3 \%$. These results compared with the results of our study show that the percentage of the small intestine is far greater. $\mathrm{V}_{45 \mathrm{~Gy}}$ in the previously mentioned study for small intestine was $10 \% . \mathrm{V}_{30 \mathrm{~Gy}}$ and $\mathrm{V}_{45 \mathrm{~Gy}}$ in our study are slightly higher, which can 
be explained by the fact that the CT simulation in the preceding study conducted in pronation and using belly board immobilization, so that most guts taken out of the target volume. In their study the way of delineation of the small intestine in terms of the volume that is being analyzed was not explained. In this study, the small intestine was delineated 1 $\mathrm{cm}$ above the PTV. In case a larger volume was delineated in their study, of course the result obtained was that a smaller volume of small intestine receives 30 and $45 \mathrm{~Gy}$. Also, in the above mentioned study it is not explained whether the delineation of the small intestine was done in a way to delineate the entire volume with associated abdominal cavity or only at intersections visible walls of the small intestine.

Guo et al. (9) published an article in which they compared the DVH parameters of three different planning techniques (conventional, conformal and IMRT). Special attention is given to the volume of the small intestine which is covered by $100 \%$ dose $\left(\mathrm{V}_{45 \mathrm{~Gy}}\right)$. For the small intestine, the conventional plan $\mathrm{V}_{45 \mathrm{~Gy}}$ was $24 \%$, while for the conformal plan it was $20 \%$. In our study $\mathrm{V}_{45 \mathrm{~Gy}}$ is higher for conventional as well as conformal plans in comparison to the results of the mentioned study. The reason for this difference lies in the fact that the mentioned conformal technique was done by using box-technique which thanks to side fields gives a minor contribution to the organs at risk. Nadeau et al. (10) compared the conventional planning with other radiation techniques. And here is a special emphasis on the volume of organs at risk which were covered by $100 \%$ dose $\left(\mathrm{V}_{45 \mathrm{~Gy}}\right)$. The mean $\mathrm{V}_{45 \mathrm{~Gy}}$ to the small intestine in the case of conventional plans amounted $45.9 \%$. The results obtained and the reasons why they differ from the results of this study can be explained in the same way as the results of the previous studies by Guo et al.

Very similar results were obtained in the study by Roeske et al (11) when, in their study, they compared the DVH parameters of conventional radiotherapy techniques with other radiation techniques. From DVH parameters for the organs at risk the volume of organ receiving 30, 40 and $45 \mathrm{~Gy}\left(\mathrm{~V}_{30 \mathrm{~Gy}}\right.$, $\mathrm{V}_{40 \mathrm{~Gy}}$ and $\mathrm{V}_{45 \mathrm{~Gy}}$ ) was observed, as well as the maximum dose on the organ at risk. $\mathrm{V}_{30 \mathrm{~Gy}}$ for the small intestine was $49.8 \pm 11.6 \%$. $\mathrm{V}_{40 G \mathrm{G}}$ for the small intestine was $40.7 \pm 10.9 \%$. and $V_{45 \mathrm{~Gy}}$ was $33.8 \pm$ $10.3 \%$. $\mathrm{D}_{\max }$ for the small intestine was $50 \mathrm{~Gy}$. The results of $\mathrm{V}_{30 \mathrm{~Gy}}, \mathrm{~V}_{40 \mathrm{~Gy}}$ and $\mathrm{V}_{45 \mathrm{G}}$ for the small intestine were higher in our study in comparison to the aforementioned study.

Parameters for 3D conformal radiotherapy of patients with endometrial cancer compared with other techniques were followed in the study conducted by Jidong Lian et al. (12). Dose contribution to the small intestine in our study is much lower in comparison to the mentioned study, which can be explained by the way of delineating the small intestine. In the aforementioned study as a "gut" they delineated the entire peritoneal cavity except liver and spleen, which significantly increased the total volume of the small intestine. Yang et al. $(13,14)$ compared the three-dimensional conformal radiotherapy techniques with other radiation techniques. The results of our studies for the small intestine are quite similar to the results in this study.

In conclusion, there is a significantly smaller dose contribution on small intestine by planning based on three-dimensional conformal radiotherapy in comparison to conventional planning. This study opens the way for further research in terms of research of inter and intra observation variations for delineation of target volume and organs at risk, errors in planning, errors in repositioning during the implementation of transcutaneous radiotherapy.

\section{COMPETING INTERESTS}

The authors declare that they have no conflict of interest.

\section{REFERENCES}

1. Cardens HR, Look K, Michael H, Careso L. Endometrium. In Halperin EC, Perez CA, Brady LW (eds). Principles and Practice of Radiation Oncology. Lipincott Williams \& Wilkins. Philadelphia, 2008th; p. 1610-1628.

2. Sutton G, Axelrod JH, Bindy BN, et al. Whole abdomen radiotherapy in the adjuvant treatment of patients with stage III and IV endometrial cancer: a Gynecological Oncology Group study. Gynecol Oncol. 2005;97:775.

3. Šamija, M. Ženski spolni sustav. U: M. Šamija,ed. Klinička onkologija. Zagreb: Medicinska naklada, 2006th; p. 266-269.

4. Yasushi N, Takehiro N, Mitsuyuki A et al. CT simulator: A new 3-D planning and simulating system for radiotherapy: Part 2. Clinical application. Int J Radiat Oncol Biol Phys. 1990;18:505-13.

5. ChaoKSC, CengizM, HerzogT Gynecologic tumors. In Gregoire V. Scalliet P. Ang KK (Eds). Clinical Target Volumes in Conformal and Intensity Modulated Radiation Therapy. Springer. Berlin, 2004th; p. 171-186.

6. Purdy JA. Current ICRU definitions of volumes: Limitations and futures directions. Semin. Radiat Oncol. 2004;14:27-40.

7. QUANTEC (Quantitative Analyses of Normal Tissue Effects in the Clinic): IJROBP, 76(2),Suppl, 2010.

8. Heron DE, Gerszten K, Selvaraj RN et al. Conventional 3D conformal ver- 
sus intensity - modulated radiotherapy for the adjuvant treatment of gynecological malignancies: A comparative dosimetric study of dose - volume histograms. Gynecol Oncol: 2003;91: 131-136

9. Guo S, Ennis D, Bhatia S, et al. Assesement of nodal target definition using three diferent techniques: implicationes for re-defining the optional pelvic field in endometrial cancer, Radiat Oncol: 2010; 5:59.

10. Nadeau S, Bouchard M, Germain I, Raymond PE, Beaulieu F, Beaulieu L, Roy R, Gingras L. Postoperative irradiation of gynecologic malignancies: improving treatment delivery using aperture-based intensity-modulated radiotherapy. Int J Radiat Oncol Biol Phys. 2007;68(2):601-11.

11. Roeske JC, Lujan A, Rotmensch J, Waggoner SE, Yamada D, Mundt AJ. Intensity-modulated whole pelvic radiation therapy in patients with gynecologic malignancies. Int J Radiat Oncol Biol Phys. 2000;48(5):1613-21.
12. Lian J, Mackenzie M, Joseph K, Pervez N, Dundas G, Urtasun R, Pearcey R. Assessment of extended-field radiotherapy for stage IIIC endometrial cancer using three-dimensional conformal radiotherapy, intensity-modulated radiotherapy, and helical tomotherapy. Int J Radiat Oncol Biol Phys. 2008;70(3):935-43.

13. Yang R, Xu S, Jiang W, Xie C, Wang J. Integral dose in three-dimensional conformal radiotherapy, intensity-modulated radiotherapy and helical tomotherapy. Clin Oncol (R Coll Radiol). 2009;21(9):706-12.

14. Yang R, Xu S, Jiang W, Wang J, Xie C. Dosimetric comparison of postoperative whole pelvic radiotherapy for endometrial cancer using threedimensional conformal radiotherapy, intensity-modulated radiotherapy, and helical tomotherapy. Acta Oncol. 2010;49(2):230-6. 